Kragujevac Journal of Mathematics

Volume 45(2) (2021), Pages 173-180.

\title{
CHEBYSHEV POLYNOMIALS FOR CERTAIN SUBCLASS OF BAZILEVIĆ FUNCTIONS ASSOCIATED WITH RUSCHEWEYH DERIVATIVE
}

\author{
ABDUL RAHMAN S. JUMA ${ }^{1}$, SABA N. AL-KHAFAJI ${ }^{2}$, AND OLGA ENGEL ${ }^{3}$ \\ ABstract. In this paper, through the instrument of the well-known Chebyshev \\ polynomials and subordination, we defined a family of functions, consisting of \\ Bazilević functions of type $\alpha$, involving the Ruscheweyh derivative operator. Also, \\ we investigate coefficient bounds and Fekete-Szegö inequalities for this class.
}

\section{INTRODUCTION AND DEFINITIONS}

Let $\mathcal{A}$ be the class of functions $f$ of the form

$$
f(z)=z+\sum_{k=2}^{\infty} a_{k} z^{k}
$$

which are analytic in the open unit disk $\mathbb{U}=\{z: z \in \mathbb{C}:|z|<1\}$. Let $\mathcal{S}$ denote the class of analytic functions $f \in \mathcal{A}$, which are univalent in $\mathbb{U}$ and are normalized with the following conditions:

$$
f(0)=0 \quad \text { and } \quad f^{\prime}(0)=1 .
$$

Let $f$ and $g$ be analytic functions in $\mathbb{U}$. We say that the function $f$ is a subordinate to $g$ in $\mathbb{U}$, written as $f \prec g$, if there exists a Schwarz function $w$, which is analytic in $\mathbb{U}$ with $w(0)=0$ and $|w(z)|<1,(z \in \mathbb{U})$ such that $f(z)=g(w(z))$. Furthermore, if $g$ is univalent in $\mathbb{U}$, then we get

$$
f(z) \prec g(z),(z \in \mathbb{U}) \Leftrightarrow f(0)=g(0) \quad \text { and } \quad f(\mathbb{U}) \subset g(\mathbb{U}) \quad(\text { see }[6]) .
$$

Key words and phrases. Analytic functions, univalent functions, Chebyshev polynomials, Ruscheweyh derivative operator, subordination, Fekete-Szegö inequalities, Bazilević functions.

2010 Mathematics Subject Classification. Primary: 30C45. Secondary: 30C50.

DOI 10.46793/KgJMat2102.173J

Received: July 05, 2018.

Accepted: November 05, 2018. 
The problem of finding the sharp bounds for the non-linear functional $\left|a_{3}-\mu a_{2}^{2}\right|$ for Taylor-Mclaurin series is popularly known as the Fekete-Szegö problem. This problem has a rich history in the geometric functions theory. Its source was in the disproof by Fekete and Szegö of the 1933 guess of Littlewood and Paley that the coefficients of odd univalent functions are limited by unity (see [7], has since received great attention, especially in many subclasses of the family of univalent functions. For that reason Fekete-Szegö functional was studied by many authors and a some assessments were found in a numerous subclasses of normalized univalent functions (see $[3,9,11,12,14]$ ).

The significance of Chebyshev polynomial in numerical analysis is increased in both theoretical and practical points of view. Out of four kinds of Chebyshev polynomials, many researchers dealing with orthogonal polynomials of Chebyshev, contain chiefly results of first and second kinds of Chebyshev polynomials $\mathrm{T}_{k}(t)$ and $\mathrm{U}_{k}(t)$ respectively and their numerous uses in different applications. Additionally, one can see those given by the papers in $([1,2,4,5]$ and $[8])$. The Chebyshev polynomials of the first and second kinds are well known in the case of a real variable $t$ on $(-1,1)$, which are defined as follows

$$
\begin{aligned}
\mathrm{T}_{k}(t) & =\cos k \theta, \\
\mathrm{U}_{k}(t) & =\frac{\sin (k+1) \theta}{\sin \theta},
\end{aligned}
$$

where $k$ is the degree of the polynomial and $t=\cos \theta$.

In [14] (also see [13]) Ruscheweyh introduced the following derivative operator:

$$
\begin{aligned}
D^{0} f(z) & =f(z), \\
D^{n} f(z) & =\frac{z\left(z^{n-1} f(z)\right)^{(n)}}{n !},
\end{aligned}
$$

for $n \in \mathbb{N}=\{1,2, \ldots\}$. The symbol $D^{n} f$ is called the $n^{\text {th }}$ order Ruscheweyh derivative of $f$.

We observe that

$$
\begin{aligned}
& D^{0} f(z)=f(z) \\
& D^{1} f(z)=z f^{\prime}(z),
\end{aligned}
$$

and in general

$$
D^{n} f(z)=z+\sum_{k=2}^{\infty} \rho(n, k) a_{k} z^{k}
$$

where

$$
\rho(n, k)=\left(\begin{array}{c}
n+k-1 \\
n
\end{array}\right)
$$


Definition 1.1. A function $f \in \mathcal{A}$ of the form (1.1) belongs to class $\mathcal{G}(\alpha, t)$, if it satisfies the following subordination

$$
\mathcal{G}(\alpha, t)=\left\{f \in \mathcal{A}: \frac{\left(D^{n} f(z)\right)^{\prime}\left(D^{n} f(z)\right)^{\alpha-1}}{z^{\alpha-1}} \prec \frac{1}{1-2 t z+z^{2}}\right\},
$$

where $0 \leq \alpha \leq 1, t \in\left(\frac{1}{2}, 1\right]$ and $z \in \mathbb{U}$.

Note that, if $t=\cos \alpha, \alpha \in(-\pi / 3, \pi / 3)$, then

$$
\begin{aligned}
H(z, t) & :=\frac{1}{1-2 \cos \alpha z+z^{2}} \\
& =1+\sum_{k=1}^{\infty} \frac{\sin ((k+1) \alpha)}{\sin \alpha} z^{k} \quad(z \in \mathbb{U}) .
\end{aligned}
$$

Thus

$$
H(z, t)=1+2 \cos \alpha z+\left(3 \cos ^{2} \alpha-\sin ^{2} \alpha\right) z^{2}+\cdots \quad(z \in \mathbb{U}) .
$$

Therefore, from [15] we can write

$$
H(z, t)=1+\mathrm{U}_{1}(t) z+\mathrm{U}_{2}(t) z^{2}+\cdots \quad(z \in \mathbb{U}, t \in(-1,1)),
$$

where

$$
\mathrm{U}_{k-1}(t)=\frac{\sin (k \arccos t)}{\sqrt{1-t^{2}}} \quad(k \in \mathbb{N})
$$

denotes the Chebyshev polynomials of the second kind. It is known that

$$
\mathrm{U}_{k}(t)=2 t \mathrm{U}_{k-1}(t)-\mathrm{U}_{k-2}(t)
$$

and

$$
\begin{aligned}
\mathrm{U}_{1}(t) & =2 t, \\
\mathrm{U}_{2}(t) & =4 t^{2}-1, \\
\mathrm{U}_{3}(t) & =8 t^{3}-4 t .
\end{aligned}
$$

The ordinary generating function for Chebyshev polynomials $\mathrm{T}_{k}(t), t \in[-1,1]$, of the first kind have the following form

$$
\sum_{k=0}^{\infty} \mathrm{T}(t) z^{k}=\frac{1-t z}{1-2 t z+z^{2}} \quad(z \in \mathbb{U})
$$

The Chebyshev polynomials of the first kinds $\mathrm{T}_{k}(t)$ and of the second kinds $\mathrm{U}_{k}(t)$ are connected by the following relations:

$$
\begin{aligned}
\frac{\mathrm{dT}_{k}(t)}{\mathrm{d} t} & =k \mathrm{U}_{k-1}(t), \\
\mathrm{T}_{k}(t) & =\mathrm{U}_{k}(t)-t \mathrm{U}_{k-1}(t), \\
2 \mathrm{~T}_{k}(t) & =\mathrm{U}_{k}(t)-\mathrm{U}_{k-2}(t) .
\end{aligned}
$$


By giving specific values to the parameters $\alpha$ and $n$ in this class we obtain the following cases.

(i) If $\alpha=0$ and $n=0$, then we get

$$
\frac{z f^{\prime}(z)}{f(z)} \prec H(z, t):=\frac{1}{1-2 t z+z^{2}},
$$

it reduces to a special case of the class $\mathcal{B}_{\Sigma}^{\mu}(\lambda, t)$, which was introduced by Bulut, Magesh and Abirami [5].

(ii) If $n=0$ and $\alpha=1$, then we get

$$
f^{\prime}(z) \prec H(z, t):=\frac{1}{1-2 t z+z^{2}},
$$

it also reduces to a special case of the class $\mathcal{B}_{\Sigma}^{\mu}(\lambda, t)$, which was introduced by Bulut, Magesh and Abirami [5].

(iii) If $n=0$, then we get

$$
f^{\prime}(z)\left(\frac{z}{f(z)}\right)^{1-\alpha} \prec H(z, t):=\frac{1}{1-2 t z+z^{2}},
$$

it reduces to the class $\mathcal{H}(\alpha)$, which was introduced by Bulut, Magesh and Abirami [5], as a special case to the class $\mathcal{B}_{\Sigma}^{\mu}(\lambda, t)$.

The aim of this paper is to provide estimates for initial coefficients of Bazilević functions of type $\alpha$ in the class $\mathcal{G}(\alpha, t)$, involving by the Ruscheweyh derivative operator. Besides that, the problem of Fekete- Szegö in this class is additionally explained.

\section{Preliminaries}

We need the following lemma to prove our main result.

Lemma 2.1 ([10]). If $w \in \mathcal{S}$, then for any complex number $\mu$

$$
\left|w_{2}-\mu w_{1}^{2}\right| \leq \max \{1,|\mu|\} .
$$

The result is sharp for the functions $w(z)=z^{2}$ or $w(z)=z$.

\section{Main Results}

Theorem 3.1. Let $f \in \mathcal{A}$ belong to the class $\mathcal{G}(\alpha, t)$. Then

$$
\left|a_{2}\right| \leq \frac{2 t}{(\alpha+1)\left(\begin{array}{c}
n+1 \\
n
\end{array}\right)}
$$


and

$$
\begin{aligned}
\left|a_{3}\right| \leq & \frac{4 t^{2}+2 t-1}{(\alpha+2)\left(\begin{array}{c}
n+2 \\
n
\end{array}\right)}+\frac{4 t^{2}}{(\alpha+1)(\alpha+2)\left(\begin{array}{c}
n+2 \\
n
\end{array}\right)}-\frac{8 \alpha t^{2}}{(\alpha+1)^{2}(\alpha+2)\left(\begin{array}{c}
n+2 \\
n
\end{array}\right)} \\
& -\frac{4 \alpha(\alpha-1) t^{2}}{2(\alpha+1)^{2}(\alpha+2)\left(\begin{array}{c}
n+1 \\
n
\end{array}\right)\left(\begin{array}{c}
n+2 \\
n
\end{array}\right)} .
\end{aligned}
$$

Proof. If $f \in \mathcal{G}(\alpha, t)$, then from (1.2) we have

$$
\frac{\left(D^{n} f(z)\right)^{\prime}\left(D^{n} f(z)\right)^{\alpha-1}}{z^{\alpha-1}}=1+\mathrm{U}_{1}(t) w(z)+\mathrm{U}_{2}(t) w^{2}(z)+\cdots .
$$

Replacing the value of $D^{n}(f(z))$ and $\left(D^{n}(f(z))\right)^{\prime}$ with their equivalent series expressions in (3.1), it follows that

$$
\frac{\left(z+\sum_{k=2}^{\infty} k \rho(n, k) a_{k} z^{k}\right)\left(1+\sum_{k=2}^{\infty} \rho(n, k) a_{k}(\alpha) z^{k-1}\right)}{z+\sum_{k=2}^{\infty} \rho(n, k) a_{k} z^{k}}=1+\mathrm{U}_{1}(t) w(z)+\mathrm{U}_{2}(t) w^{2}(z)+\cdots .
$$

By using the binomial expansion of $1+\sum_{k=2}^{\infty} \rho(n, k) a_{k}(\alpha) z^{k-1}$, upon simplification we obtain

$$
\begin{aligned}
& \left(z+2\left(\begin{array}{c}
n+1 \\
n
\end{array}\right) a_{2} z^{2}+3\left(\begin{array}{c}
n+2 \\
n
\end{array}\right) a_{3} z^{3}+\cdots\right) \\
& \times\left(1+\alpha\left(\begin{array}{c}
n+1 \\
n
\end{array}\right) a_{2} z+\left[\alpha\left(\begin{array}{c}
n+2 \\
n
\end{array}\right) a_{3}+\frac{\alpha(\alpha-1)}{2 !}\left(\begin{array}{c}
n+1 \\
n
\end{array}\right) a_{2}^{2}\right] z^{2}+\cdots\right) \\
= & {\left[1+\mathrm{U}_{1}(t) w(z)+\mathrm{U}_{2}(t) w^{2}(z)+\cdots\right]\left(z+\left(\begin{array}{c}
n+1 \\
n
\end{array}\right) a_{2} z^{2}+\left(\begin{array}{c}
n+2 \\
n
\end{array}\right) a_{3} z^{3}+\cdots\right), }
\end{aligned}
$$

where $w$ is an analytic function, such that $w(0)=0$ and

$$
|w(z)|=\left|c_{1} z+c_{2} z^{2}+c_{3} z^{3}+\cdots\right|<1 \quad(z \in \mathbb{U}),
$$

where

$$
\left|c_{j}\right| \leq 1 \quad(j \in \mathbb{N})
$$

From (3.2) and (3.3), we have

$$
\begin{aligned}
& \left(z+2\left(\begin{array}{c}
n+1 \\
n
\end{array}\right) a_{2} z^{2}+3\left(\begin{array}{c}
n+2 \\
n
\end{array}\right) a_{3} z^{3}+\cdots\right) \\
& \left.\times\left(1+\alpha\left(\begin{array}{c}
n+1 \\
n
\end{array}\right) a_{2} z+\left[\begin{array}{c}
n+2 \\
n
\end{array}\right) a_{3}+\frac{\alpha(\alpha-1)}{2 !}\left(\begin{array}{c}
n+1 \\
n
\end{array}\right) a_{2}^{2}\right] z^{2}+\cdots\right) \\
= & {\left[1+\mathrm{U}_{1}(t) c_{1} z+\left(\mathrm{U}_{1}(t) c_{2}+\mathrm{U}_{2}(t) c_{1}^{2}\right) z^{2}+\cdots\right] } \\
& \times\left(z+\left(\begin{array}{c}
n+1 \\
n
\end{array}\right) a_{2} z^{2}+\left(\begin{array}{c}
n+2 \\
n
\end{array}\right) a_{3} z^{3}+\cdots\right) .
\end{aligned}
$$


From (3.4), we obtain

$$
a_{2}=\frac{\mathrm{U}_{1}(t) c_{1}}{(\alpha+1)\left(\begin{array}{c}
n+1 \\
n
\end{array}\right)}
$$

It is easily seen that from (1.3) and (3.5), we have

$$
\left|a_{2}\right| \leq \frac{2 t}{(\alpha+1)\left(\begin{array}{c}
n+1 \\
n
\end{array}\right)}
$$

Now, in order to find the bound on $\left|a_{3}\right|$, from (9), we get

$$
\begin{aligned}
a_{3}= & \frac{\mathrm{U}_{1}(t) c_{2}+\mathrm{U}_{2}(t) c_{1}^{2}}{(\alpha+2)\left(\begin{array}{c}
n+2 \\
n
\end{array}\right)}+\frac{\mathrm{U}_{1}^{2}(t) c_{1}^{2}}{(\alpha+1)(\alpha+2)\left(\begin{array}{c}
n+2 \\
n
\end{array}\right)}-\frac{2 \alpha \mathrm{U}_{1}^{2}(t) c_{1}^{2}}{(\alpha+1)^{2}(\alpha+2)\left(\begin{array}{c}
n+2 \\
n
\end{array}\right)} \\
& -\frac{\alpha(\alpha-1) \mathrm{U}_{1}^{2}(t) c_{1}^{2}}{2(\alpha+1)^{2}(\alpha+2)\left(\begin{array}{c}
n+1 \\
n
\end{array}\right)\left(\begin{array}{c}
n+2 \\
n
\end{array}\right)} .
\end{aligned}
$$

By using (1.3) and (3.5) in (3.6), we get

$$
\begin{aligned}
\left|a_{3}\right| \leq & \frac{4 t^{2}+2 t-1}{(\alpha+2)\left(\begin{array}{c}
n+2 \\
n
\end{array}\right)}+\frac{4 t^{2}}{(\alpha+1)(\alpha+2)\left(\begin{array}{c}
n+2 \\
n
\end{array}\right)}-\frac{8 \alpha t^{2}}{(\alpha+1)^{2}(\alpha+2)\left(\begin{array}{c}
n+2 \\
n
\end{array}\right)} \\
& -\frac{4 \alpha(\alpha-1) t^{2}}{2(\alpha+1)^{2}(\alpha+2)\left(\begin{array}{c}
n+1 \\
n
\end{array}\right)\left(\begin{array}{c}
n+2 \\
n
\end{array}\right)} .
\end{aligned}
$$

Theorem 3.2. If function $f$ of the form (1.1) belongs to the class $\mathcal{G}(\alpha, t)$, then

$$
\begin{aligned}
\left|a_{3}-\mu a_{2}^{2}\right| \leq & \frac{2 t}{\left(\alpha^{2}+2\right)\left(\begin{array}{c}
n+2 \\
n
\end{array}\right)} \max \left\{1, \mid \frac{4 t^{2}-1}{2 t}+\frac{2 t}{\left(\alpha^{2}+1\right)}-\frac{4 \alpha^{2} t}{\left(\alpha^{2}+1\right)}\right. \\
& \left.-\frac{\alpha(\alpha-1) t\left(\begin{array}{c}
n+2 \\
n
\end{array}\right)}{\left(\alpha^{2}+1\right)\left(\begin{array}{c}
n+1 \\
n
\end{array}\right)^{2}}-\mu \frac{2 t\left(\begin{array}{c}
n+2 \\
n
\end{array}\right)\left(\alpha^{2}+2\right)}{\left(\alpha^{2}+1\right)^{2}\left(\begin{array}{c}
n+1 \\
n
\end{array}\right)} \mid\right\} .
\end{aligned}
$$

The result is sharp.

Proof. From (3.5) and (3.6), we get

$$
\begin{aligned}
a_{3}-\mu a_{2}^{2}= & \frac{\mathrm{U}_{1}(t) c_{2}+\mathrm{U}_{2}(t) c_{1}^{2}}{(\alpha+2)\left(\begin{array}{c}
n+2 \\
n
\end{array}\right)}+\frac{\mathrm{U}_{1}^{2}(t) c_{1}^{2}}{(\alpha+1)(\alpha+2)\left(\begin{array}{c}
n+2 \\
n
\end{array}\right)}-\frac{2 \alpha \mathrm{U}_{1}^{2}(t) c_{1}^{2}}{(\alpha+1)^{2}(\alpha+2)\left(\begin{array}{c}
n+2 \\
n
\end{array}\right)} \\
& -\frac{\alpha(\alpha-1) \mathrm{U}_{1}^{2}(t) c_{1}^{2}}{2(\alpha+1)^{2}(\alpha+2)\left(\begin{array}{c}
n+1 \\
n
\end{array}\right)\left(\begin{array}{c}
n+2 \\
n
\end{array}\right)}-\mu \frac{\mathrm{U}_{1}^{2}(t) c_{1}^{2}}{(\alpha+1)^{2}\left(\begin{array}{c}
n+1 \\
n
\end{array}\right)}
\end{aligned}
$$


Therefore,

$$
\begin{aligned}
a_{3}-\mu a_{2}^{2}= & \frac{\mathrm{U}_{1}(t)}{(\alpha+2)\left(\begin{array}{c}
n+2 \\
n
\end{array}\right)}\left[c_{2}+\left(\frac{\mathrm{U}_{2}(t)}{\mathrm{U}_{1}(t)}+\frac{\mathrm{U}_{1}(t)}{(\alpha+1)}-\frac{2 \alpha^{2} \mathrm{U}_{1}(t)}{(\alpha+1)^{2}}\right.\right. \\
& \left.\left.-\frac{\alpha(\alpha-1) \mathrm{U}_{1}(t)\left(\begin{array}{c}
n+2 \\
n
\end{array}\right)}{2(\alpha+1)^{2}\left(\begin{array}{c}
n+1 \\
n
\end{array}\right)^{2}}-\mu \frac{\mathrm{U}_{1}(t)\left(\begin{array}{c}
n+2 \\
n
\end{array}\right)(\alpha+2)}{(\alpha+1)^{2}\left(\begin{array}{c}
n+1 \\
n
\end{array}\right)^{2}}\right) c_{1}^{2}\right] .
\end{aligned}
$$

Then, in view of Lemma 2.1, we conclude that

$$
\begin{aligned}
\left|a_{3}-\mu a_{2}^{2}\right| \leq & \frac{\mathrm{U}_{1}(t)}{(\alpha+2)\left(\begin{array}{c}
n+2 \\
n
\end{array}\right)} \max \left\{1, \mid \frac{\mathrm{U}_{2}(t)}{\mathrm{U}_{1}(t)}+\frac{\mathrm{U}_{1}(t)}{(\alpha+1)}-\frac{2 \alpha^{2} \mathrm{U}_{1}(t)}{(\alpha+1)^{2}}\right. \\
& \left.-\frac{\alpha(\alpha-1) \mathrm{U}_{1}(t)\left(\begin{array}{c}
n+2 \\
n
\end{array}\right)}{2(\alpha+1)^{2}\left(\begin{array}{c}
n+1 \\
n
\end{array}\right)^{2}}-\mu \frac{\mathrm{U}_{1}(t)\left(\begin{array}{c}
n+2 \\
n
\end{array}\right)(\alpha+2)}{(\alpha+1)^{2}\left(\begin{array}{c}
n+1 \\
n
\end{array}\right)} \mid\right\},
\end{aligned}
$$

which is equivalent to

$$
\begin{aligned}
\left|a_{3}-\mu a_{2}^{2}\right| \leq & \frac{2 t}{(\alpha+2)\left(\begin{array}{c}
n+2 \\
n
\end{array}\right)} \max \left\{1, \mid \frac{4 t^{2}-1}{2 t}+\frac{2 t}{\alpha+1}-\frac{4 \alpha^{2} t}{(\alpha+1)^{2}}\right. \\
& \left.-\frac{\alpha(\alpha-1) t\left(\begin{array}{c}
n+2 \\
n
\end{array}\right)}{(\alpha+1)^{2}\left(\begin{array}{c}
n+1 \\
n
\end{array}\right)^{2}}-\mu \frac{2 t\left(\begin{array}{c}
n+2 \\
n
\end{array}\right)(\alpha+2)}{(\alpha+1)^{2}\left(\begin{array}{c}
n+1 \\
n
\end{array}\right)} \mid\right\} .
\end{aligned}
$$

This completes the proof.

Putting $\alpha=1$ in Theorem 3.2, we obtain the following result.

Corollary 3.1. If $f$ given by (1.1) belongs to the class $\mathcal{G}(1, t)$, then

$$
\left|a_{3}-\mu a_{2}^{2}\right| \leq \frac{2 t}{3\left(\begin{array}{c}
n+2 \\
n
\end{array}\right)} \max \left\{1,\left|\frac{4 t^{2}-1}{2 t}-t-\mu \frac{3 t\left(\begin{array}{c}
n+2 \\
n
\end{array}\right)}{2\left(\begin{array}{c}
n+1 \\
n
\end{array}\right)}\right|\right\} \text {. }
$$

The result is sharp.

\section{REFERENCES}

[1] S. Altinkaya and S. Y. Cin, On the chebyshev polynomial bounds for classes of univalent functions, Khayyam J. Math. 2 (2016), 1-5.

[2] S. Altinkaya and S. Y. Cin, Estimates on coefficients of a general subclass of bi-univalent functions associated with symmetric q-derivative operator by means of the chebyshev polynomials, Asia Pacific Journal of Mathematics 4 (2017), 90-99.

[3] S. Altinkaya and S. Y. Cin, On the Chebyshev polynomial coefficient problem of some subclasses of bi-univalent functions, Gulf J. Math. 5 (2017), 34-40.

[4] L. A. R. Bucur and D. Breaz, Coefficient bounds and Fekete-Szegö problem for a class of analytic functions defined by using a new differential operator, Appl. Math. Sci. 9 (2015), 1355-1368. 
[5] N. M. S. Bulut and C. Abirami, A comprehensive class of analytic bi-univalent functions by means of Chebyshev polynomials, J. Fract. Calc. Appl. 8 (2017), 32-39.

[6] N. M. S. Bulut and V. Balaji, Initial bounds for analytic and bi-univalent functions by means of Chebyshev polynomials, J. Class. Anal. 11 (2017), 83-89.

[7] P. Duren, Univalent Functions, Grundlehren der Mathematischen, Wissenschaften 259, SpringerVerlag, New York, Berlin, Tokyo, 2001.

[8] R. El-Ashwah and S. Kanas, Fekete-Szegö inequalities for quasi-subordination functions classes of complex order, Kyungpook Math. J. 55 (2015), 679-688.

[9] R. R. J. Dziok and J. Sokól, Application of chebyshev polynomials to classes of analytic functions, C. R. Math. Acad. Sci. Paris 353 (2015), 433-438.

[10] M. Fekete and G. Szegö, Eine bemerkung uber ungerade schlichte funktionen, J. Lond. Math. Soc. 2 (1933), 85-89.

[11] H. Güney, Initial Chebyshev polynomial cofficient bound estimates for bi-univalent functions, Acta Univ. Apulensis Math. Inform. (2016), 159-165.

[12] F. Keogh and E. Merkes, A coefficient inquality for certain classes of analytic functions, Proc. Amer. Math. Soc. (1969), 8-12.

[13] S. Ruscheweyh, New criteria for univalent functions, Proc. Amer. Math. Soc. (1975), 109-115.

[14] S. Ruscheweyh, Neighborhoods of univalent functions, Proc. Amer. Math. Soc. (1981), 521-527.

[15] E. Whittaker and G. Watson, A course of modern analysis. an introduction to the general theory of infinite processes and of analytic functions, ZAMM - Journal of Applied Mathematics and Mechanics 43 (1963), 435.

\author{
${ }^{1}$ Department of Mathematics, \\ UNIVERSITY OF ANBAR, \\ RAMADI-IRAQ \\ Email address: dr_juma@hotmail.com \\ ${ }^{2}$ Department of Mathematics, Faculty of Computer Science and Mathematics, \\ UNIVERSITY OF KUFA, \\ IRAQ \\ Email address: sabanf.mc11p@uokufa.edu.iq \\ ${ }^{3}$ Department of Mathematics, \\ BABEŞ-Bolyai University, \\ ROMANIA \\ Email address: engel_olga@hotmail.com
}

